Trauma Surgery \& Acute Care Open

\title{
Novel method of delivery of continuous positive airway pressure for apnea testing during brain death evaluation
}

\author{
Jennifer L Hubbard, ${ }^{1}$ Rachel C Dirks, ${ }^{1}$ Wade L Veneman, ${ }^{2}$ James W Davis ${ }^{1}$
}

${ }^{1}$ Department of Surgery, UCSF Fresno, Fresno, California, USA ${ }^{2}$ Department of Respiratory Care Services, Community Regional Medical Center, Fresno, California, USA

\section{Correspondence to} Dr Jennifer L Hubbard, Department of Surgery, University of California San Francisco-Fresno, 2823 Fresno Street, 1st Floor, Fresno, CA 93721, USA; jhubbard@fresno.ucsf. edue000046

Received 13 September 2016 Revised 22 November 2016 Accepted 25 November 2016
To cite: Hubbard JL, Dirks RC, Veneman WL, et al. Trauma Surg Acute Care Open Published Online First: [please include Day Month Year] doi:10.1136/ tsaco-2016-000046

\section{ABSTRACT}

Background There are several methods for apnea testing for the evaluation of neurological death, including oxygen via T-piece, oxygen cannula inserted into the endotracheal tube, and continuous positive airway pressure (CPAP). Lung suitability for transplantation is determined in part by the partial pressure of arterial oxygen $\left(\mathrm{PaO}_{2}\right)$ to fraction of inspired oxygen ( $\left.\mathrm{FiO}_{2} ; \mathrm{P}: \mathrm{F}\right)$ ratio. We hypothesized that delivery of CPAP during apnea testing using a novel method would improve post-test $P: F$ ratios.

Methods A retrospective review was performed at a level I trauma center for all patients undergoing apnea testing from 2010 to 2016. The CPAP system used a flow-inflating bag and was made available in 2012. It was used at the discretion of the clinician. Patients were classified as having an apnea test by CPAP or by nonCPAP method (T-piece, oxygen cannula in endotracheal tube, etc). The two groups were compared for baseline characteristics and the primary outcome of postapnea test P:F ratio.

Results During the study period, 145 patients underwent apnea testing; 67 patients by the CPAP method and 78 by non-CPAP method. There were no significant differences in demographics, mechanism of brain injury, pneumonia rate, smoking status, or antibiotic usage between the two groups. The pretest $P$ : $F$ ratio was similar between groups, but the CPAP group had significantly higher post-test P:F ratio (304 vs 250 , $p=0.02$ ). There were no reported complications arising from CPAP use.

Conclusions We describe a novel method of delivering CPAP by a flow-inflating bag during examination for brain death. This method led to improved oxygenation, P:F ratios, and may decrease barotrauma. The flowinflating bag was inexpensive, easily implemented, and without adverse effects. Multicentered, prospective trials are needed to elicit significant benefit in lung donation and transplantation.

Level of evidence Level IV, diagnostic tests.

\section{BACKGROUND}

Organ shortages continue to grow in the USA. As of August, 2016, there were over 77000 people on the active waiting list for organ transplantation. ${ }^{1}$ This great need for organs demands that as many organs as possible are procured from potential donors. $^{2}$ One of the organs with the lowest rate of recovery is lungs. In 2015, there were 9079 deceased donors. Of these, only 3791 lungs (left and right counted separately) were recovered, compared with 16408 kidneys and 7416 livers. ${ }^{3}$ Essentially all lung transplants come from deceased donors $;{ }^{1}$ therefore, management of deceased donors may greatly influence the quality of these organs.

Apnea testing is an essential component of declaration of death by neurological criteria, ${ }^{4-6}$ the prerequisite for donation by deceased donors. In their most recent guidelines, the American Academy of Neurology recommends that the apnea testing component of declaration of death by neurological criteria be performed by inserting an oxygen cannula into the trachea or using a T-piece for the maintenance of oxygenation. ${ }^{6}$ Unfortunately, disconnecting the ventilator and using an oxygen cannula or T-piece leads to atelectasis. For those patients who go on to become organ donors, atelectasis and higher pressures necessary to recruit the collapsed alveoli induced by apnea testing can cause significant injury and potentially decrease lung recovery rates. ${ }^{7}$ The objective measure of lung quality used by most transplant surgeons is the P:F ratio, the ratio of the partial pressure of arterial oxygen $\left(\mathrm{PaO}_{2}\right)$ to fraction of inspired oxygen $\left(\mathrm{FiO}_{2}\right)$. The P:F ratio has been found to be significantly associated with organ recipient prognosis ${ }^{8}$ and most transplant surgeons require a P:F ratio of $>300$ on a positive end expiratory pressure (PEEP) of 5 for eligibility. ${ }^{9}$

Continuous positive airway pressure (CPAP) delivers a constant pressure in the airways, either by invasive or non-invasive routes. CPAP has been shown to decrease atelectasis, increase P:F ratio, and decrease dyspnea in patients with respiratory compromise. ${ }^{10}$ The potential benefit of decreased atelectasis with CPAP can also be used during apnea testing. CPAP was shown to improve oxygenation in a small trial where three methods (CPAP, oxygen cannula in trachea, and T-piece) were used in each patient, ${ }^{11}$ and in a separate case report. ${ }^{12}$ For the current study, we hypothesized that apnea testing with the use of CPAP would improve post-test P:F ratios and potentially lung transplantation rates.

\section{METHODS}

Prior to 2012, there was no protocol for apnea testing at Community Regional Medical Center, a 650-bed, American College of Surgeons (ACS)-verified level 1 trauma center in Fresno, California. The medical and surgical intensivists variously used a T-piece with high flow oxygen, with the high flow oxygen cannula placed within the endotracheal tube, or CPAP via the ventilator at the discretion of the attending physician. The use of CPAP was anecdotally noted to cause less physiological disruption to the patient, but was 
complicated by the automatic ventilator's safety feature giving breaths after prolonged periods of apnea $(60 \mathrm{~s})$. In conjunction with our respiratory care practitioners (RCPs), we developed a novel method of delivering CPAP via a flow inflating bag (figure 1). The flow inflating bag, also called an anesthesia bag, only inflates when connected to a gas source. CPAP is accomplished by an adjustable flow control valve and the opening must be sealed to sustain CPAP, that is, connected to the endotracheal tube. The gas used in this case was $100 \%$ oxygen. Most clinicians are more familiar with this device being used for neonates and children, but it can also be applied in the adult population. This method was made widely available throughout our multispecialty intensive care units in 2012.

Subsequently, a retrospective review was performed for all patients undergoing apnea testing from January, 2010 to March, 2016. The study was approved by the regional organ procurement organization (OPO) Institutional Review Board (IRB) and deemed exempt by the University of California San Francisco Fresno IRB. The CPAP protocol, made available in 2012, was used at the discretion of the clinician, although encouraged by department leaders. Patients were classified as having an apnea test by CPAP or by non-CPAP method (T-piece, oxygen cannula in endotracheal tube, etc). The two groups were compared for baseline characteristics including demographics, mechanism of brain injury, torso trauma, pneumonia rate, smoking status, antibiotic usage, and ventilator mode. The primary outcomes were postapnea test $\mathrm{P}: \mathrm{F}$ ratio and lung transplantation rates. Categorical data were analyzed by Fisher's exact or $\chi^{2}$ tests and continuous variables analyzed by Mann-Whitney $U$ tests with significance attributed to a $\mathrm{p}$ value $\leq 0.05$. Data were collected from the hospital's electronic medical records as well as records supplied by our regional OPO.

\section{RESULTS}

During the study period, 145 patients underwent apnea testing; 67 patients by the CPAP method and 78 by non-CPAP method. There were no significant differences in demographics, mechanism of brain injury, smoking status, pneumonia rate, torso trauma, or antibiotic usage between the two groups (table 1). There was also no difference in the incidence of pneumonia, (community-acquired

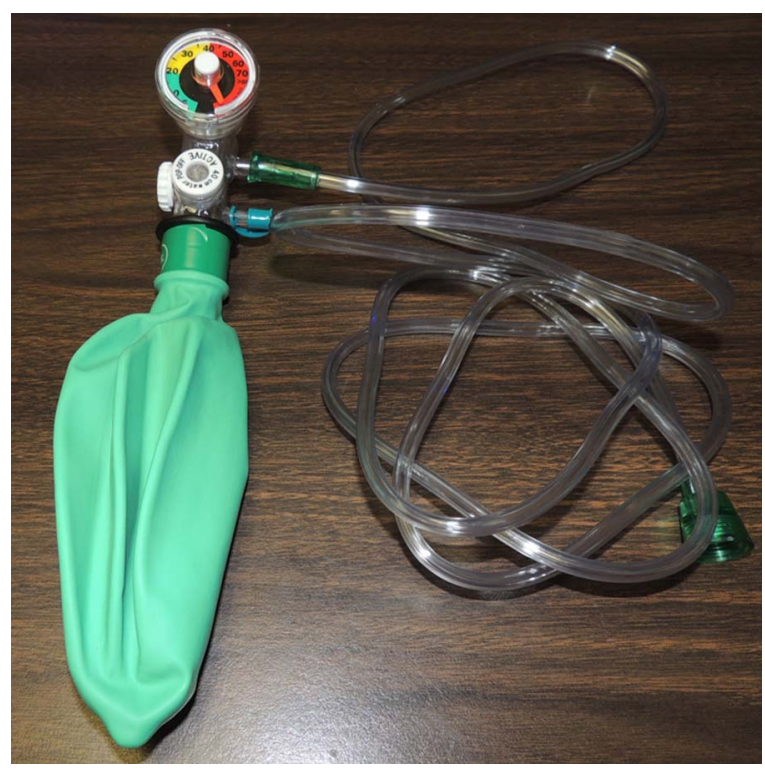

Figure 1 Flow inflating bag with adjustable pressure dial.
Table 1 Baseline characteristics

\begin{tabular}{|c|c|c|c|}
\hline & $\begin{array}{l}\text { CPAP } \\
(n=67)\end{array}$ & $\begin{array}{l}\text { T-piece } / \mathrm{O}_{2} \\
\text { cannula }(\mathrm{n}=78)\end{array}$ & $p$ Value \\
\hline Age $(m e a n \pm S D)$ & $41 \pm 15$ & $41 \pm 18$ & 0.81 \\
\hline Male gender & $49(73 \%)$ & $53(68 \%)$ & 0.50 \\
\hline \multicolumn{4}{|l|}{ Mechanism } \\
\hline Penetrating trauma & $13(19 \%)$ & $16(21 \%)$ & 0.87 \\
\hline Blunt trauma & $25(37 \%)$ & $22(28 \%)$ & 0.24 \\
\hline Stroke & $21(31 \%)$ & $32(41 \%)$ & 0.23 \\
\hline Anoxia & $6(9 \%)$ & $8(10 \%)$ & 0.79 \\
\hline Other & $2(3 \%)$ & 0 & 0.21 \\
\hline \multicolumn{4}{|l|}{ Smoking history } \\
\hline Yes & $7(10 \%)$ & $6(8 \%)$ & 0.92 \\
\hline No & $20(30 \%)$ & $16(20 \%)$ & $\begin{array}{l}\text { (for } \\
\text { known }\end{array}$ \\
\hline Unknown & $40(60 \%)$ & $56(72 \%)$ & history) \\
\hline Pulmonary contusion & $14(21 \%)$ & $6(8 \%)$ & 0.022 \\
\hline Rib fractures & $6(9 \%)$ & $3(4 \%)$ & 0.30 \\
\hline Clinical pneumonia & $8(12 \%)$ & $5(6 \%)$ & 0.25 \\
\hline \multicolumn{4}{|l|}{ Respiratory cultures } \\
\hline Negative & $26(39 \%)$ & $28(36 \%)$ & 0.72 \\
\hline Community-acquired* & $20(30 \%)$ & $21(27 \%)$ & 0.70 \\
\hline Hospital-acquiredt & $10(15 \%)$ & $10(13 \%)$ & 0.71 \\
\hline Not done & $11(16 \%)$ & $19(24 \%)$ & 0.24 \\
\hline \multicolumn{4}{|l|}{ Antibiotics } \\
\hline None & $50(75 \%)$ & $55(71 \%)$ & 0.58 \\
\hline Narrow spectrum & $5(7 \%)$ & $4(5 \%)$ & 0.73 \\
\hline Broad spectrum & $12(18 \%)$ & $19(24 \%)$ & 0.35 \\
\hline \multicolumn{4}{|l|}{ Clinician performing test } \\
\hline Surgical & $35(52 \%)$ & $41(53 \%)$ & 0.97 \\
\hline Medical & $30(45 \%)$ & $34(44 \%)$ & 0.89 \\
\hline Neurosurgical & $2(3 \%)$ & $3(4 \%)$ & 0.78 \\
\hline Days postadmit (mean) & $2.9 \pm 2.8$ & $2.7 \pm 2.5$ & 0.47 \\
\hline $\begin{array}{l}\text { Donor management with APRV (\% } \\
\text { of authorized donors) }\end{array}$ & $11(23 \%)$ & $3(5 \%)$ & 0.009 \\
\hline
\end{tabular}

organisms or healthcare facility-acquired organisms) or admission service (medical vs surgical). There was a higher rate of pulmonary contusions in the CPAP group ( $21 \%$ vs $8 \%, p=0.022)$.

Table 2 demonstrates the outcomes measured between the two groups. Pretest ventilator modes, oxygenation, and P:F ratios were similar between groups. The CPAP group versus non-CPAP group had significantly higher post-test P:F ratio (304 vs $250, p=0.02$ ). The CPAP group had longer length of tests that approached statistical significance $(11.1$ vs $10.4 \mathrm{~min}$, $\mathrm{p}=0.052$ ). Additionally, the percentage of lung donors was higher in the CPAP group, although the power was not sufficient to obtain statistical significance. There were no reported complications arising from CPAP use.

\section{DISCUSSION}

Organ shortages throughout the USA continue to be problematic. One way to increase the number of organs available is to maximize the number and quality of organs from each donor. This requires meticulous care of the donor while they are still a living patient, through the process of declaration of death by neurological criteria, and after declaration of death, when the 


\section{Table 2 Outcomes}

\begin{tabular}{|c|c|c|c|}
\hline & $\begin{array}{l}\text { CPAP } \\
(\mathrm{n}=67)\end{array}$ & $\begin{array}{l}\text { T-piece/ } / \mathrm{O}_{2} \text { cannula } \\
(\mathrm{n}=78)\end{array}$ & p Value \\
\hline Pretest vent mode APRV & $1(1 \%)$ & $4(5 \%)$ & 0.37 \\
\hline Pretest $\mathrm{PaO}_{2}$ & $128 \pm 63$ & $130 \pm 67$ & 0.93 \\
\hline Pretest P:F & $316 \pm 97$ & $331 \pm 120$ & 0.65 \\
\hline End-of-test P:F & $304 \pm 135$ & $250 \pm 143$ & 0.02 \\
\hline Post-test compliance $\left(\mathrm{mL} / \mathrm{cm} \mathrm{H}{ }_{2} \mathrm{O}\right)$ & $41 \pm 17$ & $40 \pm 11$ & 0.77 \\
\hline Length of test (min) & $11.1 \pm 2.2$ & $10.4 \pm 2.4$ & 0.052 \\
\hline Post-test vent mode APRV & $2(3 \%)$ & $2(3 \%)$ & 1.00 \\
\hline Next day P:F & $296 \pm 117$ & $297 \pm 122$ & 0.87 \\
\hline Authorization for organ donation & $47(70 \%)$ & $57(73 \%)$ & 0.70 \\
\hline $\begin{array}{l}\text { Lungs transplanted (\% of } \\
\text { authorized donors) }\end{array}$ & $15(32 \%)$ & $13(23 \%)$ & 0.30 \\
\hline
\end{tabular}

OPO takes over care of the patient. Our organization routinely uses lung-protective strategies with low tidal volumes $(5-8 \mathrm{~mL} /$ $\mathrm{kg}$ ) as this was shown to improve lung transplantation rates in a prior randomized trial. ${ }^{13}$ However, our lung retrieval and transplantation rates were still suboptimal. In this study, we describe a technique which minimizes atelectasis by using CPAP for apnea testing for declaration of death by neurological criteria in order to prevent further injury to the lungs.

When an apneic patient is disconnected from the ventilator circuit for even brief periods of time, atelectasis, or collapse of the alveoli occurs. Once atelectasis occurs, greater pressure is required to reopen the airway than to maintain expansion of an already open airway. ${ }^{14}$ Therefore, maintaining expansion of the alveoli during a prolonged period of apnea should lead to decreased pressure needed to re-expand the airway and less barotrauma to the airways. When we first took on this quality improvement initiative, it seemed easiest to use the ventilator in a CPAP mode. However, ventilators have a safety over-ride that initiates a breath after prolonged periods of apnea. The clinicians could determine that it was not a patient-initiated breath, but it confounds the overall validity of the test, as well as causing further confusion to family members who were present for the test. Using a flow inflating bag eliminated the possibility of an over-ride mechanism, yet remained inexpensive and simple to use.

Implementation of the CPAP protocol in our hospital was straightforward. The training for the RCPs took place as part of their monthly mandatory training sessions. The overall use of the device is simple and was easily implemented. When a physician notified the RCP that they were going to perform an apnea test, the RCP suggested use of the flow-inflating bag method. Over time, more physicians have adopted this method as its use has demonstrated good results and improved oxygenation. The flow inflating bag is equipment that is already available in most acute care hospitals, particularly in the emergency department, operating room, or in areas caring for children and neonates. The overall cost of the apparatus is $\$ 10-\$ 20$, limiting additional cost to the patient and hospital. The technique is now used by all clinicians in our institution. It has become our standard of care. Since the system is quite simple and effective, no changes have been made since it was instituted.

Although there was no statistical difference noted in most of the outcomes, the experience of the clinicians using this method was uniformly positive. Anecdotally, we were able to perform apnea tests for longer periods of time with little or no oxygen desaturation. Saturations during the apnea test are not recorded in the medical record and the length of the test is documented inconsistently, so we were unable to statistically analyze these variables. We also anecdotally noted that we were able to complete apnea tests on patients with worse pulmonary function using the CPAP method, as was similarly noted in a prior case report. $^{12}$

Interestingly, as noted in table 2, the end-of-test P:F ratio was significantly higher in the CPAP group. Furthermore, several patients actually had higher $\mathrm{PaO}_{2}$ at the end of the test than they did at the beginning of the test. Feedback from the OPO was that the lung quality was significantly better in patients undergoing apnea testing with the CPAP method. The following day P:F ratios returned to essentially equivalent values, likely reflecting the ability of the lung to overcome the atelectasis. The amount of barotrauma re-expansion causes is unknown, but may play a role in the overall quality of the lungs. Unfortunately, this did not translate into a significant increase in the number of lungs transplanted during the study period. While we cannot ascertain the exact cause, it is likely a combination of factors such as an underpowered study in combination with clinical factors used in consideration of suitability for transplantation. A larger, randomized study might be able to assess these factors fully.

There are limitations to our study. The sample size is small and outcomes such as lung recovery rate are affected by a myriad of factors beyond a single procedure. As with any retrospective study, the possibility of selection bias exists. Prior to institution of the CPAP method, the method of apnea testing was always left to the treating physician. This continued to be the case after the method was instituted, though the RCPs and department leaders encouraged the switch. This was confirmed in reviewing the patient records. It appears that the selection of test method was based more on physician preference and not patient characteristics. This is reinforced by the similarity of the baseline characteristics between groups. A further confounding factor is the increased use of airway pressure release ventilation (APRV) in the CPAP group. In small series, APRV has been shown to improve lung retrieval rates. ${ }^{15}$ After further investigation, we found that the vast majority of patients who had use of APRV were on it for $<6$ hours. The increased use of APRV in the CPAP group also likely reflects a shift in management strategies over time. The CPAP method was employed more frequently in the later time periods. This coincided with a shift in OPO management that included the use of APRV for short periods of time for selected patients. Further studies are needed to delineate the optimum protocols for management of donors prior to declaration of death by neurological criteria and OPO management after declaration.

In conclusion, apnea testing using CPAP has only been reported in a case report and a small series in the literature and has never been reported using a flow-inflating bag method. In this novel pilot study, we found that use of CPAP for apnea testing improved P:F ratios. This method was inexpensive, easily implemented, and without adverse effects. Further investigations with multicentered, prospective trials are warranted to elicit a true benefit in outcomes.

Acknowledgements Significant portions of data were provided by Donor Network West. The authors sincerely appreciate their help and support with this project. National statistics and data was supported in part by Health Resources and Services Administration contract 234-2005-37011C.

Contributors JLH and WLV designed the protocol and the study and did the data collection. RCD performed the statistical analysis. All authors participated in data interpretation. JLH wrote the manuscript, which all authors critically revised.

Disclaimer The content is the responsibility of the authors alone and does not necessarily reflect the views or policies of the Department of Health and Human 
Services, nor does mention of trade names, commercial products, or organizations imply endorsement by the US Government.

Competing interests None declared.

\section{Patient consent Obtained.}

Ethics approval Local ethics committee in each of the participating centers.

Provenance and peer review Not commissioned; externally peer reviewed.

Data sharing statement The only unpublished data are various clinical findings immediately before, during, and after apnea testing. Only the authors have access to the data.

Open Access This is an Open Access article distributed in accordance with the Creative Commons Attribution Non Commercial (CC BY-NC 4.0) license, which permits others to distribute, remix, adapt, build upon this work non-commercially, and license their derivative works on different terms, provided the original work is properly cited and the use is non-commercial. See: http://creativecommons.org/ licenses/by-nc/4.0/

\section{REFERENCES}

1 http://www.unos.org/ last (accessed 18 Aug 2016).

2 United States Organ Transplantation. OPTN \& SRTR Annual Data Report, 2011. US Department of Health and Human Services. Health Resources and Services Administration, 2012

3 https://optn.transplant.hrsa.gov/data/view-data-reports/national-data/ Donors Recovered in the US by Donor Type, OPTN data; last (accessed 18 Aug 2016).

4 Sharpe MD, Young GB, Harris $C$. The apnea test for brain death determination: an alternative approach. Neurocrit Care 2004;1:363-6.

5 Drazkowski J. Determining brain death: back to the basics. Semin Neurol 2007;27:393-9.

6 Wijdicks EF, Varelas PN, Gronseth GS, Greer DM, American Academy of Neurology. Evidence-based guideline update: determining brain death in adults: report of the
Quality Standards Subcommittee of the American Academy of Neurology. Neurology 2010;74:1911-18.

7 Paries M, Boccheciampe N, Raux M, Riou B, Langeron O, Nicolas-Robin A. Benefit of a single recruitment maneuver after an apnea test for the diagnosis of brain death. Crit Care 2012;16:R116.

8 Thabut G, Mal H, Cerrina J, Dartevelle P, Dromer C, Velly JF, Stern M, Loirat P, Bertocchi M, Mornex JF, et al. Influence of donor characteristics on outcome after lung transplantation: a multicenter study. J Heart Lung Transplant 2005;24:1347-53.

9 Snell Gl, Westall GP. Selection and management of the lung donor. Clin Chest Med 2011;32:223-32.

10 Cammarota G, Vaschetto R, Turucz E, Dellapiazza F, Colombo D, Blando C, Della Corte F, Maggiore SM, Navalesi P. Influence of lung collapse distribution on the physiologic response to recruitment maneuvers during noninvasive continuous positive airway pressure. Intensive Care Med 2011;37:1095-102.

11 Lévesque S, Lessard MR, Nicole PC, Langevin S, LeBlanc F, Lauzier F, Brochu JG. Efficacy of a T-piece system and a continuous positive airway pressure system for apnea testing in the diagnosis of brain death. Crit Care Med 2006:34:2213-16.

12 Shrestha GS, Shrestha PS, Acharya SP, Sedain G, Bhandari S, Aryal D, Gajurel B, Marhatta MN, Amatya R. Apnea testing with continuous positive airway pressure for the diagnosis of brain death in a patient with poor baseline oxygenation status. Indian J Crit Care Med 2014;18:331-3.

13 Mascia L, Pasero D, Slutsky AS, Arguis MJ, Berardino M, Grasso S, Munari M, Boifava S, Cornara G, Della Corte F, et al. Effect of a lung protective strategy for organ donors on eligibility and availability of lungs for transplantation: a randomized controlled trial. JAMA 2010;304:2620-7.

14 Bendixen HH, Hedley-Whyte J, Laver MB. Impaired oxygenation in surgical patients during general anesthesia with controlled ventilation. A concept of atelectasis. N Engl J Med 1963;269:991-6.

15 Hanna K, Seder CW, Weinberger JB, Sills PA, Hagan M, Janczyk RJ. Airway pressure release ventilation and successful lung donation. Arch Surg 2011;146:325-8. 